\title{
EIGENVALUES FOR A COMBINATION BETWEEN LOCAL AND NONLOCAL $p$-LAPLACIANS
}

\author{
LEANDRO M. DEL PEZZO, RAÚL FERREIRA, AND JULIO D. ROSSI
}

ABSTRACT. In this paper we study the Dirichlet eigenvalue problem

$$
\begin{cases}-\Delta_{p} u-\Delta_{J, p} u=\lambda|u|^{p-2} u & \text { in } \Omega, \\ u=0 & \text { in } \Omega^{c}=\mathbb{R}^{N} \backslash \Omega .\end{cases}
$$

Here $\Delta_{p} u$ is the standard local $p$-Laplacian, $\Delta_{J, p} u$ is a nonlocal, $p$-homogeneous operator of order zero and $\Omega$ is a bounded domain in $\mathbb{R}^{N}$.

We show that the first eigenvalue (that is isolated and simple) satisfies $\left(\lambda_{1}\right)^{1 / p} \rightarrow \Lambda$ as $p \rightarrow \infty$ where $\Lambda$ can be characterized in terms of the geometry of $\Omega$. We also find that the eigenfunctions converge, $u_{\infty}=\lim _{p \rightarrow \infty} u_{p}$, and find the limit problem that is satisfied in the limit.

\section{INTRODUCTION}

Eigenvalue problems for the $p$-Laplacian is by now a classical subject. In fact, the study of eigenvalues, that is, nontrivial solutions to

$$
-\Delta_{p} u=-\operatorname{div}\left(|\nabla u|^{p-2} \nabla u\right)=\lambda|u|^{p-2} u
$$

in a bounded smooth domain, $\Omega$, with Dirichlet boundary conditions $u=0$ on $\partial \Omega$ starts with [15] (we refer to [23] and references therein on the $p$-Laplacian). For this eigenvalue problem it is known that there exists a sequence $\lambda_{n} \rightarrow \infty$ of eigenvalues, that the first (the smallest) eigenvalue is isolated and simple and has a positive associated eigenfunction. The description of the complete spectrum remains as a mayor open problem. There are two extreme cases that deserved special attention, $p=1$ (related to the Cheeger problem, see [24]) and $p=\infty$ (this case is understood as the limit as $p \rightarrow \infty$, see $[18,19])$. The analysis of this kind of problems goes far beyond this brief description, including eigenvalues with weights, variable exponents, systems, etc.

On the other hand, recently, there is an increasing interest in the study of nonlocal operators, like the fractional $p$-Laplacian. For references concerning nonlocal fractional problems we refer to $[7,20,22,12]$ and references therein. For the eigenvalue problem for this operator we refer to $[6,9,10,11,13,22,14]$ where a detailed study was carry over showing similarities and differences with the local case (one of the biggest differences is that the restriction of an eigenfunction to a nodal domain is not an eigenfunction of this nodal domain due to the nonlocal character of the problem). Also in [22] the limit case $p \rightarrow \infty$ was studied in the fractional setting. Concerning nonlocal operators of zero-order, that is, problems of the form

$$
-\int_{\mathbb{R}^{N}} J(x-y)|u(x)-u(y)|^{p-2}(u(y)-u(x)) d y=\lambda|u|^{p-2} u
$$

involving non-singular and compactly supported kernels (with $u=0$ in $\mathbb{R}^{N} \backslash \Omega$ ) we refer to $[3,5]$ where it is shown that the constant that corresponds to the infimum of the corresponding Raleigh quotient is strictly positive. However, it is not known that it is attained due to the lack of compactness. 
The purpose of this paper is to study the following Dirichlet eigenvalue problem

$$
\begin{cases}\mathcal{L}_{J, p}(u)=\lambda|u|^{p-2} u & \text { in } \Omega, \\ u=0 & \text { in } \Omega^{c}:=\mathbb{R}^{N} \backslash \Omega .\end{cases}
$$

Here $\Omega$ be a bounded connected domain in $\mathbb{R}^{N}$ with $C^{1, \alpha}$ boundary, $p \geq 2$ and

$$
\mathcal{L}_{J, p}(u):=-\Delta_{p} u-\Delta_{J, p} u
$$

where

$$
\Delta_{p}(u):=\operatorname{div}\left(|\nabla u|^{p-2} \nabla u\right)
$$

is the usual local $p$-Laplacian operator and

$$
\Delta_{J, p} u:=2 \int_{\mathbb{R}^{N}} J(x-y)|u(x)-u(y)|^{p-2}(u(y)-u(x)) d y,
$$

where the kernel $J: \mathbb{R}^{N} \rightarrow \mathbb{R}$ is a radially symmetric, nonnegative continuous function with compact support, $J(0)>0$ and $\int_{\mathbb{R}^{N}} J(x) d x=1$, (this last condition is imposed just for normalization). Since we assumed $J$ to be continuous and compactly supported we let

$$
R_{J}:=\inf \left\{R: J(x)=0 \text { in } \mathbb{R}^{N} \backslash B(0, R)\right\} \in(0,+\infty),
$$

and we also assume that $J(x)>0$ for $|x|<R_{J}$.

A scalar $\lambda$ is called an eigenvalue of $\mathcal{L}_{J, p}$ if there is a nontrivial weak solution $u \in W_{0}^{1, p}(\Omega)$ of $(1)$, that is, a function $u$ such that

$$
\mathcal{H}_{J, p}(u, v)=\lambda \int_{\Omega}|u|^{p-2} u v d x \quad \forall v \in W_{0}^{1, p}(\Omega),
$$

holds. Here

$$
\begin{aligned}
\mathcal{H}_{J, p}(u, v):= & \int_{\Omega}|\nabla u|^{p-2} \nabla u \nabla v d x \\
& +\int_{\mathbb{R}^{N}} \int_{\mathbb{R}^{N}} J(x-y)|u(x)-u(y)|^{p-2}(u(x)-u(y))(v(x)-v(y)) d x d y .
\end{aligned}
$$

Such $u$ is is called an eigenfunction corresponding to the eigenvalue $\lambda$.

From the usual variational techniques we have that the first (the smallest) eigenvalue of (1) is

$$
\lambda_{1}(p):=\inf \left\{\frac{\mathcal{H}_{J, p}(u, u)}{\|u\|_{p}^{p}}: u \in W_{0}^{1, p}(\Omega) \backslash\{0\}\right\},
$$

where $\|\cdot\|_{p}$ denotes the $L^{p}(\Omega)$-norm.

Moreover, using a topological tool (the genus), we can construct a sequence of eigenvalues $\left\{\lambda_{k}\right\}_{k \in \mathbb{N}}$ of (1) such that $\lambda_{k} \nearrow \infty$ as $k \rightarrow \infty$.

Theorem 1.1. The value $\lambda_{1}(p)$ is the first eigenvalue of (1) (if $\lambda$ is an eigenvalue of (1) then $\left.\lambda \geq \lambda_{1}(p)\right)$. In addition $\lambda_{1}(p)$ is simple and isolated, and its corresponding eigenfunctions have constant sign.

Moreover, there exists a sequence of eigenvalues $\left\{\lambda_{k}\right\}_{k \in \mathbb{N}}$ such that $\lambda_{k} \nearrow \infty$ as $k \rightarrow \infty$. Every eigenfunction $u$ verifies that $u \in C^{1, \alpha}(\bar{\Omega})$ for some $\alpha \in(0,1)$.

Now, we deal with the limit as $p \rightarrow \infty$.

Theorem 1.2. It holds that

$$
\lim _{p \rightarrow \infty}\left[\lambda_{1}(p)\right]^{1 / p}=\Lambda
$$


where

with

$$
\Lambda:=\inf \left\{\frac{\max \left\{\|\nabla u\|_{\infty},[u]_{J}\right\}}{\|u\|_{\infty}}: u \in W_{0}^{1, \infty}(\Omega)\right\}
$$

$$
[u]_{J}:=\sup \{|u(x)-u(y)|: x-y \in \operatorname{supp}(J)\} .
$$

Moreover, let $u_{p}$ be a nonnegative eigenfunction corresponding to the first eigenvalue $\lambda_{1}(p)$ and normalize it by $\left\|u_{p}\right\|_{p}=1$. Then, there is a sequence $\left\{p_{n}\right\}_{n \in \mathbb{N}}$ such that $p_{n} \rightarrow \infty, u_{p_{n}} \rightarrow u_{\infty} \in W_{0}^{1, \infty}(\Omega)$ uniformly in $\bar{\Omega}$ and it holds that

$$
\Lambda=\frac{\max \left\{\left\|\nabla u_{\infty}\right\|_{\infty},\left[u_{\infty}\right]_{J}\right\}}{\left\|u_{\infty}\right\|_{\infty}} .
$$

This limit value $\Lambda$ can be characterized in terms of the geometry of the domain $\Omega$. In fact, in terms of $R_{J}$ and the inradius (the radius of the largest ball contained in $\Omega$ ), that is

$$
R_{\Omega}:=\max \{\operatorname{dist}(x, \partial \Omega): x \in \bar{\Omega}\} .
$$

For the characterization of $\Lambda$ we introduce $K_{\Omega} \in \mathbb{N} \cup\{0\}$ and $b \in\left[0, R_{J}\right)$ as

$$
R_{\Omega}=K_{\Omega} R_{J}+b,
$$

that is, we define $K_{\Omega}$ as the quotient and $b$ as the remainder of the division between $R_{\Omega}$ and $R_{J}$.

We distinguish four cases.

(1) If $b=0$ then

$$
\Lambda=\max \left\{\frac{1}{R_{\Omega}}, \frac{1}{K_{\Omega}}\right\} .
$$

(2) If $K_{\Omega}=0$ or $K_{\Omega} \neq 0$ with $b \neq 0$ and $R_{J} \leq 1$ then

$$
\Lambda=\max \left\{\frac{1}{R_{\Omega}}, \frac{1}{K_{\Omega}+1}\right\} .
$$

(3) If $K_{\Omega}>0, R_{J}>1$ and $b \geq 1$ then

$$
\Lambda=\frac{1}{K_{\Omega}+1} \text {. }
$$

(4) Finally, if $K_{\Omega}>0,1>b>0$ and $R_{J}>1$ then

$$
\Lambda=\frac{1}{K_{\Omega}+b} \text {. }
$$

Remark that in this characterization of the limit of the first eigenvalue appears in a nontrivial way the interplay between the two operators involved. The proof is based in a careful choice of test functions for the infimum that defines $\Lambda$. In these test function one can see the local/nonlocal character of the limit problem.

Concerning the equation verified by the limit we introduce the following operators

$$
\begin{gathered}
\Delta_{\infty} u=\sum_{i, j=1}^{N} u_{x_{i}} u_{x_{j}} u_{x_{i} x_{j}}=\left\langle D^{2} u \nabla u, \nabla u\right\rangle, \\
\mathcal{L}_{J, \infty} u=\sup _{x-y \in \operatorname{supp}(J)}(u(y)-u(x))+\inf _{x-y \in \operatorname{supp}(J)}(u(y)-u(x)) .
\end{gathered}
$$

Following ideas in [4], this operator can be decomposed as follows

$$
\mathcal{L}_{J, \infty} u=\mathcal{L}_{J, \infty}^{+} u+\mathcal{L}_{J, \infty}^{-} u
$$


where

$$
\mathcal{L}_{J, \infty}^{+} u=\sup _{x-y \in \operatorname{supp}(J)}(u(y)-u(x)) \quad \text { and } \quad \mathcal{L}_{J, \infty}^{-} u=\inf _{x-y \in \operatorname{supp}(J)}(u(y)-u(x)) .
$$

Theorem 1.3. Any uniform limit of eigenfunctions $u_{p}$ corresponding to the first eigenvalue $\lambda_{1}(p)$, is a viscosity solution to

$$
\begin{cases}\max \left\{M_{1}(u), M_{2}(u)\right\}=0 & \text { in } \Omega \\ u=0 & \text { on } \partial \Omega\end{cases}
$$

where

$$
M_{1}(u(x)):=\min \left\{-\Lambda u(x)-\mathcal{L}_{J, \infty}^{-} u(x),-\mathcal{L}_{J, \infty} u(x),-\mathcal{L}_{\infty, J}^{-} u(x)-|\nabla u(x)|\right\}
$$

and

$$
\begin{gathered}
M_{2}(u(x)):=\min \left\{|\nabla u(x)|-\Lambda u(x),-\Delta_{\infty} u(x),|\nabla u(x)|-\mathcal{L}_{\infty, J}^{+} u(x),\right. \\
\left.|\nabla u(x)|+\mathcal{L}_{\infty, J}^{-} u(x)\right\}
\end{gathered}
$$

Notice that the equation $\max \left\{M_{1}(u), M_{2}(u)\right\}=0$ is a sort of eigenvalue problem (the value $\Lambda$ appears there and if $u$ is a viscosity solution then $k u$ is also a solution for every $k>0)$. In addition, we highlight the local/nonlocal character of $M_{1}(u)$ and $M_{2}(u)$.

Remark 1.1. Here we assumed that $p \geq 2$. Some of our results can be extended to $1<p<2$ but in this case the equation is singular (one of the main difficulties that we found is to establish the validity of the strong maximum principle for $1<p<2$, see Lemma 2.2 for $p \geq 2$ ). Also, notice that for $1<p<2$ we need to be more careful in the definition of viscosity solutions, see [17] for the $p$-Laplace equation.

Remark 1.2. Our results can be extended to cover the problems

$$
\begin{cases}-\Delta_{p}^{s} u-\Delta_{J, p} u=\lambda|u|^{p-2} u & \text { in } \Omega \\ u=0 & \text { in } \Omega^{c} .\end{cases}
$$

and

$$
\begin{cases}-\Delta_{p} u-\Delta_{p}^{s} u=\lambda|u|^{p-2} u & \text { in } \Omega \\ u=0 & \text { in } \Omega^{c}\end{cases}
$$

In fact, we have

$$
\left[\lambda_{1}(p)\right]^{1 / p} \rightarrow \Lambda
$$

where

$$
\Lambda:= \begin{cases}\inf \left\{\frac{\max \left\{[u]_{s},[u]_{J}\right\}}{\|u\|_{\infty}}: u \in W_{0}^{1, \infty}(\Omega)\right\} & \text { for (2), } \\ \inf \left\{\frac{\max \left\{\|\nabla u\|_{\infty},[u]_{s}\right\}}{\|u\|_{\infty}}: u \in W_{0}^{1, \infty}(\Omega)\right\} & \text { for (3). }\end{cases}
$$

Here, $[u]_{s}$ is the $s$-Hölder seminorm,

$$
[u]_{s}:=\sup _{x, y \in \mathbb{R}^{N}}\left\{\frac{|u(x)-u(y)|}{|x-y|^{s}}\right\} .
$$

We present our results for (1) since it involves a combination of operators in the extreme cases, a local operator and a zero-order one. 
Remark 1.3. If we consider (for two parameters $\alpha, \beta>0$ )

$$
\begin{cases}-\alpha \Delta_{p} u-\beta \Delta_{J, p} u=\lambda|u|^{p-2} u & \text { in } \Omega, \\ u=0 & \text { in } \Omega^{c},\end{cases}
$$

one can easily show that the first eigenvalue for this problem, $\lambda_{1}(p, \alpha, \beta)$ verifies similar properties as the ones described here for the case $\alpha=\beta=1$. Moreover, it holds that

$$
\lim _{\alpha \rightarrow 1, \beta \rightarrow 0} \lambda_{1}(p, \alpha, \beta)=\lambda_{1} \quad \text { and } \quad \lim _{\alpha \rightarrow 0, \beta \rightarrow 1} \lambda_{1}(p, \alpha, \beta)=\lambda_{1, J}
$$

where $\lambda_{1}$ is the first eigenvalue for the $p$-Laplacian and $\lambda_{1, J}$ is the infimum of the corresponding Raleigh quotient for the nonlocal zero order operator.

Concerning eigenfunctions we have

$$
\lim _{\alpha \rightarrow 1, \beta \rightarrow 0} u_{1}(p, \alpha, \beta)=u_{1}
$$

with $u_{1}$ an eigenfunction of the $p$-Laplacian associated to $\lambda_{1}$. However, one can not

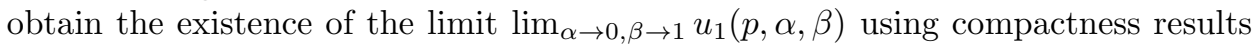
since in this case there is no uniform bound for $\|\nabla u\|_{p}$.

Therefore, one can look at (4) as an eigenvalue problem that interpolates between a local operator and a zero-order one.

\section{Some General RESUlts}

2.1. Boundary regularity. Let $\Omega$ be a bounded domain in $\mathbb{R}^{N}$ with $C^{1, \alpha}$ boundary, $K, M_{0}$ be a positive constants and $a: \bar{\Omega} \times \mathbb{R} \times \mathbb{R}^{N} \rightarrow \mathbb{R}$ be such that

$$
|a(x, z, l)| \leq K(1+|l|)^{p+2}
$$

for all $(x, z, l)$ in $\partial \Omega \times\left[-M_{0}, M_{0}\right] \times \mathbb{R}^{N}$. Before setting our regularity result, we introduce our definition of weak solution to

$$
\begin{cases}\mathcal{L}_{J, p}(u)=a(x, u, \nabla u) & \text { in } \Omega, \\ u=0 & \text { in } \Omega^{c} .\end{cases}
$$

Definition 2.1. A weak solution of (5) is a function $u \in W_{0}^{1, p}(\Omega)$ such that

$$
\mathcal{H}_{J, p}(u, v)=\int_{\Omega} a(x, u, \nabla u) v d x \quad \forall v \in W_{0}^{1, p}(\Omega)
$$

where

$$
\begin{aligned}
\mathcal{H}_{J, p}(u, v):= & \int_{\Omega}|\nabla u|^{p-2} u \nabla v d x \\
& +\int_{\mathbb{R}^{N}} \int_{\mathbb{R}^{N}} J(x-y)|u(x)-u(y)|^{p-2}(u(x)-u(y))(v(x)-v(y)) d x d y .
\end{aligned}
$$

Observe that if $u \in L^{\infty}\left(\mathbb{R}^{N}\right)$, then $\Delta_{J, p} u \in L^{\infty}\left(\mathbb{R}^{N}\right)$. Therefore, as a consequence of [21, Theorem 1] we have our regularity result.

Theorem 2.1. If $u$ is a bounded weak solution of (5) with $\|u\|_{L^{\infty}(\Omega)} \leq M_{0}$ then there is a positive constant $\alpha$ such that $u \in C^{1, \alpha}(\bar{\Omega})$. 
2.2. Comparison principle. Given $u, v \in W^{1, p}\left(\mathbb{R}^{N}\right)$, we say that

$$
\mathcal{L}_{J, p} u \leq \mathcal{L}_{J, p} v \text { in } \Omega,
$$

in the weak sense, if

$$
\mathcal{H}_{J, p}(u, w) \leq \mathcal{H}_{J, p}(v, w) \quad \forall w \in W_{0}^{1, p}(\Omega), w \geq 0 .
$$

Lemma 2.1 (Comparison principle). If $u, v \in W^{1, p}\left(\mathbb{R}^{N}\right)$ and

$$
\mathcal{L}_{J, p} u \leq \mathcal{L}_{J, p} v \quad \text { in } \Omega,
$$

in the weak sense, and $u \leq v$ in $\Omega^{c}$ then $u \leq v$ in $\Omega$.

Proof. By (6), for all $w \in W_{0}^{1, p}(\Omega), w \geq 0$ we have that

$$
\begin{aligned}
0 \leq & \int_{\Omega}\left(|\nabla v|^{p-2} \nabla v-|\nabla u|^{p-2} \nabla u\right) \nabla w d x \\
& +\int_{\mathbb{R}^{N}} \int_{\mathbb{R}^{N}} J(x-y) \mathcal{A}(v, u, x, y)(w(x)-w(y)) d x d y
\end{aligned}
$$

where

$$
\mathcal{A}(v, u, x, y)=|v(x)-v(y)|^{p-2}(v(x)-v(y))-|u(x)-u(y)|^{p-2}(u(x)-u(y)) .
$$

Now, we choice $w=\max \{u-v, 0\}$ that is nonnegative and belongs to $W_{0}^{1, p}(\Omega)$ by hypothesis. Arguing as [22, Proof of Lemma 9], we have

$$
\int_{\mathbb{R}^{N}} \int_{\mathbb{R}^{N}} J(x-y) \mathcal{A}(v, u, x, y)(w(x)-w(y)) d x d y \leq 0 .
$$

On the other hand, by Cauchy-Schwarz and Young inequalities, we have

$$
\int_{\Omega}\left(|\nabla v|^{p-2} \nabla v-|\nabla u|^{p-2} \nabla u\right) \nabla w d x \leq 0 .
$$

Therefore

$$
\begin{array}{r}
\int_{\Omega}\left(|\nabla v|^{p-2} \nabla v-|\nabla u|^{p-2} \nabla u\right) \nabla w d x=0, \\
\int_{\mathbb{R}^{N}} \int_{\mathbb{R}^{N}} J(x-y) \mathcal{A}(v, u, x, y)(w(x)-w(y)) d x d y=0 .
\end{array}
$$

Then by $\left[25\right.$, See (2.2)], we have that $w \equiv 0$ and therefore $v \geq u$ in $\mathbb{R}^{N}$.

Corollary 2.1 (Weak maximum principle). If $u \in W^{1, p}\left(\mathbb{R}^{N}\right)$ and

$$
\mathcal{L}_{J, p} u \geq 0 \quad \text { in } \Omega,
$$

in the weak sense and $u \geq 0$ in $\Omega^{c}$ then $u \geq 0$ in $\Omega$.

2.3. Viscosity solutions. Now we introduce our definition of viscosity solution of

$$
\begin{cases}\mathcal{L}_{J, p} u=f(x) & \text { in } \Omega, \\ u=0 & \text { in } \Omega^{c} .\end{cases}
$$

Here $f: \Omega \rightarrow \mathbb{R}$ is a continuous function.

Definition 2.2. Let $f: \Omega \rightarrow \mathbb{R}$ be a continuous function and $p \geq 2$. A upper semicontinuous function $u: \mathbb{R}^{N} \rightarrow[-\infty,+\infty)$ is a viscosity subsolution of $(7)$ if

(i) $u$ is not identically $-\infty$. 
(ii) For any open set $U \subset \Omega$, any $x_{0} \in U$ and any $\phi \in C^{2}(U)$ such that $\phi\left(x_{0}\right)=$ $u\left(x_{0}\right)$ and $\phi \geq u$ in $U$ if we let

we have that

$$
v(x)= \begin{cases}\phi(x) & \text { if } x \in U, \\ u(x) & \text { if } x \in U^{c},\end{cases}
$$

(iii) $u \leq 0$ in $\Omega^{c}$.

$$
\mathcal{L}_{J, p} v\left(x_{0}\right) \leq f\left(x_{0}\right)
$$

Similarly, a lower semicontinuous function $u: \mathbb{R}^{N} \rightarrow(-\infty,+\infty]$ is a viscosity supersolution of (7) if

(i) $u$ is not identically $+\infty$.

(ii) For any open set $U \subset \Omega$, any $x_{0} \in U$ and any $\psi \in C^{2}(U)$ such that $\psi\left(x_{0}\right)=$ $u\left(x_{0}\right)$ and $\psi \leq u$ in $U$ if we let

we have that

$$
w(x)= \begin{cases}\psi(x) & \text { if } x \in U, \\ u(x) & \text { if } x \in U^{c}\end{cases}
$$

$$
\mathcal{L}_{J, p} w\left(x_{0}\right) \geq f\left(x_{0}\right)
$$

(iii) $u \geq 0$ in $\Omega^{c}$.

Finally, a continuous function $u$ is a viscosity solution of (7) if it is both a viscosity subsolution and a viscosity supersolution.

Observe that if $u$ is a bounded weak solution of (7) with $f \in L^{\infty}(\Omega)$, from our previous regularity result, Theorem 2.1 , we have that $u$ is continuous in $\mathbb{R}^{N}$. Then it makes sense to ask if a weak solution is also a viscosity solution. In fact, following [19] (see also [22, Section 4] for a nonlocal counterpart), we can show the following result.

Theorem 2.2. Let $f \in C(\bar{\Omega})$. If $u \in W^{1, p}(\Omega) \cap L^{\infty}(\Omega)$ is a weak solution of $(7)$ then $u$ is a viscosity solution of (7).

Proof. Once we know that $u$ is continuous and write the problem as

$$
\begin{cases}-\Delta_{p}^{s} u=\Delta_{J, p} u+\lambda|u|^{p-2} u & \text { in } \Omega, \\ u=0 & \text { in } \Omega^{c},\end{cases}
$$

the result follows exactly as in [19] (remark that the right hand side is continuous as a function of $x \in \Omega$ ).

To conclude, we set a strong maximum principle for viscosity supersolution.

Lemma 2.2 (Strong maximum principle). Let $p \geq 2$. If $u$ is a viscosity supersolution of

$$
\begin{cases}\mathcal{L}_{J, p} u=0 & \text { in } \Omega, \\ u=0 & \text { in } \Omega^{c}\end{cases}
$$

then $u=0$ in $\mathbb{R}^{N}$ or $u>0$ in $\Omega$.

Proof. Without loss of generality we can assume $u \geq 0$ in $\mathbb{R}^{\mathbb{N}}$. Suppose that there is $x_{0} \in \Omega$ such that $u\left(x_{0}\right)=0$. Given $\varepsilon>0$ such that $\varepsilon<R_{J}$ and $B\left(x_{0}, \varepsilon\right) \subset \Omega$, we take

$$
w(x)= \begin{cases}\psi(x) & \text { if } x \in B\left(x_{0}, \varepsilon\right), \\ u(x) & \text { if } x \in B\left(x_{0}, \varepsilon\right)^{c} .\end{cases}
$$


Observe that $w(x) \leq u(x)$ for all $x \in \mathbb{R}^{N}$. Then, using that $u$ is a viscosity supersolution of $(8)$ we have that

$$
0 \leq \mathcal{L}_{J, p} w\left(x_{0}\right) \leq-\int_{\mathbb{R}^{N}} J\left(x_{0}-y\right) w(y)^{p-1} d y .
$$

Since $J$ is nonnegative, we get $w(x)=0$ for all $x \in\left\{x_{0}\right\}-\operatorname{supp}(J)$. Therefore $u(x)=0$ in $B\left(x_{0}, R_{J}\right) \backslash B\left(x_{0}, \varepsilon\right)$. Moreover, as $\varepsilon$ is arbitrary small, we get $u(x)=0$ in $B\left(x_{0}, R_{J}\right)$.

If we repeat this procedure, since $\Omega$ is a bounded connected domain, we can obtain an open cover $\left\{U_{n}\right\}_{n=1}^{m}$ of $\Omega$ such that $u=0$ in $U_{n}$ for all $n \in\{1, \ldots, m\}$, that is $u=0$ in $\Omega$.

\section{Eigenvalues}

We start this section by showing that any eigenfunction is bounded.

Lemma 3.1. If $u$ is an eigenfunction corresponding to the eigenvalue $\lambda$ then $u \in$ $L^{\infty}(\Omega)$.

Proof. If $p>N$, by the Sobolev embedding theorem we have that $u \in L^{\infty}(\Omega)$. To extend the result to the case $1<p \leq N$, we will follow ideas from [14].

Observe that it is enough to prove that $u_{+}:=\max \{u, 0\} \in L^{\infty}(\Omega)$ since $-u$ is also an eigenfunction corresponding to $\lambda$. In fact, since (1) is homogeneous, it is enough to prove that

$$
\left\|u_{+}\right\|_{\infty}:=\left\|u_{+}\right\|_{L^{\infty}(\Omega)} \leq 1 \quad \text { if }\left\|u_{+}\right\|_{p} \leq \delta
$$

where $\delta>0$ we will determined.

For all $n \in \mathbb{N}$ we define

$$
w_{n}(x):=\left(u-\left(1-2^{-n}\right)\right)_{+} .
$$

Observe that $w_{n} \in W_{0}^{1, p}(\Omega)$. Then using that

- $\left|\nabla w_{n}(x)\right|^{p}=|\nabla u(x)|^{p-2} \nabla u(x) \nabla w_{n}(x)$ a.e. in $\mathbb{R}^{N}$,

- For any $v \in W_{0}^{1, p}(\Omega)$,

$|v(x)-v(y)|^{p-2}(v(x)-v(y))\left(v_{+}(x)-v_{+}(y)\right) \geq\left|v_{+}(x)-v_{+}(y)\right|^{p} \geq 0$,

- $u$ is an eigenfunction corresponding to $\lambda$,

we have that

$$
\int_{\Omega}\left|\nabla w_{n+1}\right|^{p} d x \leq \mathcal{H}_{J, p}\left(u, w_{n+1}\right)=\lambda \int_{\Omega}|u|^{p-2} u w_{n+1} d x .
$$

The rest of the proof is entirely similar to that of [14, Theorem 3.2].

Thus, by Lemma 3.1 and Theorem 2.1 we get

Corollary 3.1. If $u$ is an eigenfunction corresponding to the eigenvalue $\lambda$ then $u \in C^{1, \alpha}(\bar{\Omega})$ for some $\alpha \in(0,1)$.

Moreover, from Corollary 3.1 and Theorem 2.2 we obtain

Corollary 3.2. If $p \geq 2$ and $u$ is an eigenfunction corresponding to the eigenvalue $\lambda$ then $u$ is a viscosity solution of (1). 
3.1. The first eigenvalue. First we show that if $u$ is an eigenfunction corresponding to $\lambda_{1}(p)$ then $u$ has constant sign.

Lemma 3.2. Let $p \geq 2$. If $u$ is an eigenfunction corresponding to $\lambda_{1}(p)$ then $u$ has constant sign.

Proof. Since $u$ is an eigenfunction corresponding to $\lambda_{1}(p)$, so is $|u|$. Therefore we can assume with no loss of generality that $u$ is nonnegative. Then, by Corollary 3.2 and Lemma 2.2, we have that $u>0$ in $\Omega$.

Our next result shows that the first eigenvalue is simple.

Theorem 3.1. Let $p \geq 2$ and fix $u$ a positive eigenfunction corresponding to $\lambda_{1}(p)$. If $\lambda>0$ is such that there is a non-negative eigenfunction $v$ corresponding to $\lambda$ then $\lambda=\lambda_{1}(p)$ and there is $k \in \mathbb{R}$ such that $v=k u$ in $\mathbb{R}^{N}$.

Proof. By Corollary 3.2 and Lemma 2.2, we have that $v>0$ in $\Omega$. Moreover for all $n \in \mathbb{N}$ we have that

$$
w_{n}:=\frac{u^{p}}{(v+1 / n)^{p-1}} \in W_{0}^{1, p}(\Omega) .
$$

Then, by Picone's identity (see [1]), we have

$$
\begin{aligned}
0 \leq & \int_{\Omega}|\nabla u|^{p}+(p-1) \frac{u^{p}}{(v+1 / n)^{p}}|\nabla v|^{p}-p \frac{u^{p-1}}{(v+1 / n)^{p-1}}|\nabla v|^{p-2} \nabla v \nabla u d x \\
= & \int\left(|\nabla u|^{p}-|\nabla v|^{p-2} \nabla v \nabla w_{n}\right) d x \\
= & \lambda_{1}(p) \int_{\Omega}|u|^{p} d x-\lambda \int_{\Omega}|v|^{p-2} v w_{n} d x \\
& \quad-\int_{\mathbb{R}^{N}} \int_{\mathbb{R}^{N}} J(x-y)|u(x)-u(y)|^{p} d x d y \\
& \quad+\int_{\mathbb{R}^{N}} \int_{\mathbb{R}^{N}} J(x-y)|v(x)-v(y)|^{p-2}(v(x)-v(y))\left(w_{n}(x)-w_{n}(y)\right) d x d y .
\end{aligned}
$$

On the other hand, by [2, Lemma 6.2$]$, we have that

$$
\begin{aligned}
& -\int_{\mathbb{R}^{N}} \int_{\mathbb{R}^{N}} J(x-y)|u(x)-u(y)|^{p} d x d y \\
& +\int_{\mathbb{R}^{N}} \int_{\mathbb{R}^{N}} J(x-y)|v(x)-v(y)|^{p-2}(v(x)-v(y))\left(w_{n}(x)-w_{n}(y)\right) d x d y \leq 0 .
\end{aligned}
$$

Therefore

$$
\begin{aligned}
0 & \leq \int_{\Omega}|\nabla u|^{p}+(p-1) \frac{u^{p}}{(v+1 / n)^{p}}|\nabla v|^{p}-p \frac{u^{p-1}}{(v+1 / n)^{p-1}}|\nabla v|^{p-2} \nabla v \nabla u d x \\
& \leq \lambda_{1}(p) \int_{\Omega}|u|^{p} d x-\lambda \int_{\Omega}|v|^{p-2} v w_{n} d x
\end{aligned}
$$

By the dominated convergence theorem and Fatou's lemma we get

$$
\begin{aligned}
0 & \leq \int_{\Omega}\left(|\nabla u|^{p}+(p-1) \frac{u^{p}}{v^{p}}|\nabla v|^{p}-p \frac{u^{p-1}}{v^{p-1}}|\nabla v|^{p-2} \nabla v \nabla u\right) d x \\
& \leq\left(\lambda_{1}(p)-\lambda\right) \int_{\Omega}|u|^{p} d x .
\end{aligned}
$$

Therefore, $\lambda=\lambda_{1}(p)$. 
Moreover, using again Picone's identity, we obtain

$$
|\nabla u|^{p}+(p-1) \frac{u^{p}}{v^{p}}|\nabla v|^{p}-p \frac{u^{p-1}}{v^{p-1}}|\nabla v|^{p-2} \nabla v \nabla u=0 \quad \text { a. e. in } \mathbb{R}^{N} .
$$

Thus, there is $k>0$ with $v=k u$ in $\Omega$.

Our next goal is to show that $\lambda_{1}(p)$ is isolated. For this we need the following technical lemma.

Lemma 3.3. Let $p \geq 2$. If $u$ is an eigenfunction corresponding to $\lambda>\lambda_{1}(p)$ then there is a positive constant $C$ independent of $u$ such that

$$
C<|\{u<0\}| \text {. }
$$

Proof. By Theorem 3.1, we have that $u_{-}:=\min \{u, 0\} \not \equiv 0$. Since $u_{-} \in W_{0}^{1, p}(\Omega)$, by the Sobolev embedding theorem for any $q \in\left(p, p_{*}\right)$ there is a constant $C$ independent of $u$ such that

$$
C\left\|u_{-}\right\|_{q} \leq\left\|\nabla u_{-}\right\|_{p} .
$$

Here $p_{*}$ denotes the Sobolev critical exponent, tha is

$$
p_{*}= \begin{cases}\frac{N p}{N-p} & \text { if } p<N \\ \infty & \text { if } p \geq N .\end{cases}
$$

Thus, by (9) we get

$$
C\left\|u_{-}\right\|_{q}^{p} \leq\left\|\nabla u_{-}\right\|_{p}^{p} \leq \mathcal{H}_{J, p}\left(u, u_{-}\right) \leq \lambda\left\|u_{-}\right\|_{L^{p}(\Omega)}^{p} .
$$

Finally, by Hölder's inequality we get

$$
C\left\|u_{-}\right\|_{p}^{p} \leq \lambda\left\|u_{-}\right\|_{q}^{p}|\{u<0\}|^{\frac{q-p}{q}} .
$$

Therefore

$$
\left(\frac{C}{\lambda}\right)^{\frac{q}{p-q}} \leq|\{u<0\}| .
$$

Now, proceeding as in the proof of [9, Theorem 4.11] we show that the next result holds.

Theorem 3.2. Let $p \geq 2$. Then $\lambda_{1}(p)$ is isolated.

Proof. By definition, $\lambda_{1}(p)$ is left-isolated. To prove that $\lambda_{1}(p)$ is right-isolated, we argue by contradiction. We assume that there is a sequence of eigenvalues $\left\{\lambda_{k}\right\}_{k \in \mathbb{N}}$ such that $\lambda_{k} \searrow \lambda_{1}(p)$ as $k \rightarrow \infty$. Let $u_{k}$ be an eigenfunction associated to $\lambda_{k}$ such that $\left\|u_{k}\right\|_{p}=1$. Then $\left\{u_{k}\right\}_{k \in \mathbb{N}}$ is bounded in $W_{0}^{s, p}(\Omega)$ and therefore we can extract a subsequence (that we still denoted by $\left\{u_{k}\right\}_{k \in \mathbb{N}}$ ) such that

$$
u_{k} \rightarrow u \text { weakly in } W_{0}^{s, p}(\Omega), \quad u_{k} \rightarrow u \text { strongly in } L^{p}(\Omega) .
$$

Then $\|u\|_{p}=1$ and $u$ is an eigenfunction associated to $\lambda_{1}(p)$. Therefore $u$ has constant sign.

Now, we can arrive to a contradiction. By Egoroff's theorem we can find a subset $A_{\delta}$ of $\Omega$ such that $\left|A_{\delta}\right|<\delta$ and $u_{k} \rightarrow u$ uniformly in $\Omega \backslash A_{\delta}$. From Lemma 3.3 and the uniform convergence in $\Omega \backslash A_{\delta}$ we obtain that $|\{u>0\}|>0$ and $|\{u>0\}|<0$. This contradicts the fact that an eigenfunction associated with the first eigenvalue does not change sign.

We also have the existence of higher eigenvalues. 
Theorem 3.3. There is a sequence of eigenvalues $\lambda_{n}$ such that $\lambda_{n} \rightarrow \infty$ as $n \rightarrow \infty$.

Proof. It follows as in [16] and hence we omit the details and only sketch the proof for the reader's convenience. Let us consider

$$
M_{\alpha}=\left\{u \in W_{0}^{1, p}(\Omega): \mathcal{H}_{J, p}(u, u)=p \alpha\right\}
$$

and

$$
\varphi(u)=\frac{1}{p} \int_{\Omega}|u|^{p} .
$$

We are looking for critical points of $\varphi$ restricted to the manifold $M_{\alpha}$ using a minimax technique. We consider the class

$$
\Sigma=\left\{A \subset W_{0}^{1, p}(\Omega) \backslash\{0\}: A \text { is closed, } A=-A\right\} .
$$

Over this class we define the genus, $\gamma: \Sigma \rightarrow \mathbb{N} \cup\{\infty\}$, as

$$
\gamma(A)=\min \left\{k \in \mathbb{N}: \text { there exists } \phi \in C\left(A, \mathbb{R}^{k}-\{0\}\right), \phi(x)=-\phi(-x)\right\} .
$$

Now, we let

$$
C_{k}=\left\{C \subset M_{\alpha}: C \text { is compact, symmetric and } \gamma(C) \leq k\right\}
$$

and let

$$
\beta_{k}=\sup _{C \in C_{k}} \min _{u \in C} \varphi(u)
$$

Then $\beta_{k}>0$ and there exists $u_{k} \in M_{\alpha}$ such that $\varphi\left(u_{k}\right)=\beta_{k}$ and $u_{k}$ is a weak eigenfuction with $\lambda_{k}=\alpha / \beta_{k}$.

\section{The limit Case $p \rightarrow \infty$}

In this section, we study the asymptotic behaviour of $\lambda_{1}(p)^{1 / p}$ as $p \rightarrow \infty$.

From now on, $u_{p}$ denotes the positive eigenfunction corresponding to $\lambda_{1}(p)$ such that $\left\|u_{p}\right\|_{p}=1$.

In order to pass to the limit in $\mathcal{H}_{J, p}(u, u)$ it is clear that for a fixed smooth $u$, it holds that $\|\nabla u\|_{p} \rightarrow\|\nabla u\|_{\infty}$. To deal with the non-local term we use that $J$ is a radially symmetric and compactly supported function to obtain

$$
\begin{aligned}
\int_{\mathbb{R}^{N}} \int_{\mathbb{R}^{N}} J(x-y)|u(x)-u(y)|^{p} d x d y= & \int_{\Omega} \int_{\Omega} J(x-y)|u(x)-u(y)|^{p} d x d y \\
& +2 \int_{\Omega} \int_{\Omega_{J} \backslash \Omega} J(x-y)|u(x)-u(y)|^{p} d x d y .
\end{aligned}
$$

where

$$
\Omega_{J}=\bigcup_{x \in \Omega}\{x-\operatorname{supp}(J)\}
$$

Let us define

$$
[u]_{J}:=\sup \{|u(x)-u(y)|: x-y \in \operatorname{supp}(J)\} .
$$

Thus, for a fixed $u$ we have

$$
\begin{aligned}
\left(\int_{\mathbb{R}^{N}} \int_{\mathbb{R}^{N}} J(x-y)|u(x)-u(y)|^{p} d x d y\right)^{1 / p} & \leq\left(2 \int_{\Omega} \int_{\Omega_{J}} J(x-y) d x d y\right)^{1 / p}[u]_{J} \\
& \rightarrow[u]_{J} \quad \text { as } p \rightarrow \infty .
\end{aligned}
$$


On the other hand, if we let $\Gamma_{\delta}=\left\{x \in \Omega, y \in \Omega_{J}: J(x-y)>\delta\right\}$, then

$$
\begin{aligned}
\left(\int_{\mathbb{R}^{N}} \int_{\mathbb{R}^{N}} J(x-y)|u(x)-u(y)|^{p} d x d y\right)^{1 / p} & \geq\left(\delta \iint_{\Gamma_{\delta}}|u(x)-u(y)|^{p} d x d y\right)^{1 / p} \\
& \rightarrow \sup _{x, y \in \Gamma_{\delta}}|u(x)-u(y)| \quad \text { as } p \rightarrow \infty \\
& \rightarrow[u]_{J} \text { as } \delta \rightarrow 0 .
\end{aligned}
$$

Hence, we obtain that

$$
\left(\int_{\mathbb{R}^{N}} \int_{\mathbb{R}^{N}} J(x-y)|u(x)-u(y)|^{p} d x d y\right)^{1 / p} \rightarrow[u]_{J} \quad \text { as } p \rightarrow \infty .
$$

Therefore, for a fixed $u$ we have

$$
\left(\mathcal{H}_{J, p}(u, u)\right)^{1 / p} \rightarrow \max \left\{\|\nabla u\|_{\infty},[u]_{J}\right\} \quad \text { as } p \rightarrow \infty .
$$

Now we are ready to prove Theorem 1.2 that says that

$$
\left[\lambda_{1}(p)\right]^{1 / p} \rightarrow \Lambda:=\inf \left\{\frac{\max \left\{\|\nabla u\|_{\infty},[u]_{J}\right\}}{\|u\|_{\infty}}: u \in W_{0}^{1, \infty}(\Omega)\right\},
$$

and that there is a sequence $\left\{p_{n}\right\}_{n \in \mathbb{N}}$ such that $p_{n} \rightarrow \infty, u_{p_{n}} \rightarrow u_{\infty}$ uniformly in $\bar{\Omega}$ with

$$
\Lambda=\frac{\max \left\{\left\|\nabla u_{\infty}\right\|_{\infty},\left[u_{\infty}\right]_{J}\right\}}{\left\|u_{\infty}\right\|_{\infty}} .
$$

Proof of Theorem 1.2. Let $u \in W_{0}^{1, \infty}(\Omega)$ be a nontrivial function. Then for any $p$ we have that $u \in W_{0}^{1, p}(\Omega) \backslash\{0\}$ and therefore

$$
\lambda_{1}(p) \leq \frac{\mathcal{H}_{J, p}(u, u)}{\|u\|_{p}^{p}} .
$$

Then, by (10),

Since $u$ is arbitrary, we get

$$
\limsup _{p \rightarrow \infty}\left[\lambda_{1}(p)\right]^{1 / p} \leq \frac{\max \left\{\|\nabla u\|_{\infty},[u]_{J}\right\}}{\|u\|_{\infty}}
$$

$$
\limsup _{p \rightarrow \infty}\left[\lambda_{1}(p)\right]^{1 / p} \leq \Lambda
$$

Our next aim is to show that

$$
\liminf _{p \rightarrow \infty}\left[\lambda_{1}(p)\right]^{1 / p} \geq \Lambda
$$

Let $\left\{p_{n}\right\}_{m \in \mathbb{N}}$ be such that $p_{n} \rightarrow \infty$ and

$$
\begin{aligned}
\liminf _{p \rightarrow \infty}[ & \left.\lambda_{1}(p)\right]^{1 / p}=\lim _{n \rightarrow \infty}\left[\lambda_{1}\left(p_{n}\right)\right]^{1 / p_{n}} \\
& =\lim _{n \rightarrow \infty}\left\|\nabla u_{p_{n}}\right\|_{p_{n}}^{p_{n}}+\int_{\mathbb{R}^{N}} \int_{\mathbb{R}^{N}} J(x-y)\left|u_{p_{n}}(x)-u_{p_{n}}(y)\right|^{p_{n}} d x d y .
\end{aligned}
$$

Observe that, from (11), we get

$$
\left\|\nabla u_{p_{n}}\right\|_{p_{n}} \leq(2 \Lambda)^{1 / p_{n}}
$$

for any $n \in \mathbb{N}$. Now, given $q>N$ there is $n_{0}$ such that $q<p_{n}$ for all $n \geq n_{0}$. Thus, using Hölder's inequality and (11), we have

$$
\left\|\nabla u_{p_{n}}\right\|_{q} \leq\left\|\nabla u_{p_{n}}\right\|_{p_{n}}|\Omega|^{1 / q-1 / p_{n}} \leq(2 \Lambda)^{1 / p_{n}}|\Omega|^{1 / q-1 / p_{n}}
$$


for all $n \geq n_{0}$. Therefore $\left\{u_{p_{n}}\right\}_{n \geq n_{0}}$ is bounded in $W_{0}^{1, q}(\Omega)$. Thus, by the Sobolev embedding theorem, passing to a subsequence, still denoted by $\left\{p_{n}\right\}_{n \in \mathbb{N}}$, we have that

weakly in $W_{0}^{1, q}(\Omega)$ and uniformly in $\bar{\Omega}$.

$$
u_{p_{n}} \rightarrow u_{\infty}
$$

On the other hand, using again Hölder's inequality, we get

$$
\begin{aligned}
& \mathcal{H}_{J, q}\left(u_{p_{n}}, u_{p_{n}}\right) \leq\left\|\nabla u_{p_{n}}\right\|_{p_{n}}^{q}|\Omega|^{1-q / p_{n}} \\
& +\left(\int_{\mathbb{R}^{N}} \int_{\mathbb{R}^{N}} J(x-y)\left|u_{p_{n}}(x)-u_{p_{n}}(y)\right|^{p_{n}} d x d y\right)^{q / p_{n}} \\
& \quad \times\left(\int_{\Omega} \int_{\Omega_{J}} J(x-y) d x d y\right)^{1-q / p_{n}} \\
& \leq\left\{|\Omega|^{1-q / p_{n}}+\int_{\Omega} \int_{\Omega_{J}} J(x-y) d x d y\right\}^{1-q / p_{n}}\left[\lambda_{1}\left(p_{n}\right)\right]^{q / p_{n}} .
\end{aligned}
$$

Passing to the limit one obtains

$$
\left[\mathcal{H}_{J, q}\left(u_{\infty}, u_{\infty}\right)\right]^{1 / q} \leq\left\{|\Omega|+\left(\int_{\Omega} \int_{\Omega_{J}} J(x-y) d x d y\right)\right\}^{1 / q} \liminf _{p \rightarrow \infty}\left[\lambda_{1}(p)\right]^{1 / p} .
$$

Observe that the above inequality holds for any $q>N$ (using a diagonal argument), and then we get that $u_{\infty} \in W_{0}^{1, \infty}(\Omega)$ and taking the limit as $q \rightarrow \infty$ in the last inequality we obtain

$$
\max \left\{\left\|\nabla u_{\infty}\right\|_{\infty},\left[u_{\infty}\right]_{J}\right\} \leq \liminf _{p \rightarrow \infty}\left[\lambda_{1}(p)\right]^{1 / p} .
$$

Moreover from the uniform convergence and the normalization condition, we get $\left\|u_{\infty}\right\|_{\infty}=1$ and therefore

$$
\Lambda \leq \max \left\{\left\|\nabla u_{\infty}\right\|_{\infty},\left[u_{\infty}\right]_{J}\right\} \leq \liminf _{p \rightarrow \infty}\left[\lambda_{1}(p)\right]^{1 / p} .
$$

Then by (11) and (12) we have that

$$
\Lambda=\lim _{p \rightarrow \infty}\left[\lambda_{1}(p)\right]^{1 / p}=\max \left\{\left\|\nabla u_{\infty}\right\|_{\infty},\left[u_{\infty}\right]_{J}\right\} .
$$

Now we will give a characterization of $\Lambda$ in terms of $R_{J}$ and the inradius (the radius of the largest ball contained in $\Omega$ ). Recall that we introduced the notation

$$
R_{\Omega}:=\max \{\operatorname{dist}(x, \partial \Omega): x \in \bar{\Omega}\},
$$

for the inradius and $K_{\Omega} \in \mathbb{N} \cup\{0\}$ and $b \in\left[0, R_{J}\right)$ as

$$
R_{\Omega}=K_{\Omega} R_{J}+b,
$$

that is, we define $K_{\Omega}$ as the quotient and $b$ as the remainder of the division between $R_{\Omega}$ and $R_{J}$.

Lemma 4.1. For $b=0$, the parameter $\Lambda$ satisfies the lower estimate

$$
\Lambda \geq \max \left\{\frac{1}{K_{\Omega}}, \frac{1}{R_{\Omega}}\right\},
$$

while for $b \neq 0$ we have the following estimate

$$
\Lambda \geq \max \left\{\frac{1}{R_{\Omega}}, \frac{1}{K_{\Omega}+b}, \frac{1}{K_{\Omega}+1}\right\} .
$$


Proof. By [18, 19], we have

$$
\frac{1}{R_{\Omega}} \leq \Lambda
$$

On the other hand, for $b=0$ it is easy to check directly that if $u \in W_{0}^{1, \infty}(\Omega)$ then

$$
\|u\|_{\infty} \leq K_{\Omega}[u]_{J} \quad R_{J}<R_{\Omega}
$$

Indeed, for all $x \in \Omega$ there exists a sequence of points $\left\{x_{i}\right\}_{i=0}^{K_{\Omega}} \in \bar{\Omega}$ with $\left|x_{i+1}-x_{i}\right| \leq$ $R_{J}$ such that $x_{0}=x$ and $x_{K_{\Omega}} \in \partial \Omega$, then

$$
|u(x)| \leq\left|u(x)-u\left(x_{1}\right)\right|+\left|u\left(x_{1}\right)-u\left(x_{2}\right)\right|+\cdots+\left|u\left(x_{K_{\Omega}-1}\right)-u\left(x_{K_{\Omega}}\right)\right| \leq K_{\Omega}[u]_{J} .
$$

Therefore,

$$
\frac{1}{K_{\Omega}} \leq \Lambda
$$

Notice that by the same argument if $b \neq 0$,

$$
\|u\|_{\infty} \leq\left(K_{\Omega}+1\right)[u]_{J}
$$

which implies

$$
\frac{1}{K_{\Omega}+1} \leq \Lambda
$$

In order to obtain the second estimate for $b \neq 0$ we note that

- If $\operatorname{dist}(x, \partial \Omega) \leq R_{J} K_{\Omega}$ then

$$
|u(x)| \leq K_{\Omega}[u]_{J} \leq K_{\Omega} \max \left\{[u]_{J},\|\nabla u\|_{\infty}\right\} ;
$$

- If $\operatorname{dist}(x, \partial \Omega)>R_{J} K_{\Omega}$ then

$$
\begin{aligned}
|u(x)| & \leq K_{\Omega}[u]_{J}+\left(\operatorname{dist}(x, \partial \Omega)-K_{\Omega} R_{J}\right)\|\nabla u\|_{\infty} \\
& \leq K_{\Omega}[u]_{J}+b\|\nabla u\|_{\infty} \\
& \leq\left(K_{\Omega}+b\right) \max \left\{[u]_{J},\|\nabla u\|_{\infty}\right\} .
\end{aligned}
$$

Thus,

$$
\frac{1}{K_{\Omega}+b} \leq \Lambda
$$

Finally, by (13), (14), (15), and (16) the result follows.

To characterize $\Lambda$ we consider four different cases.

Theorem 4.1. If $b=0$ then

$$
\Lambda=\max \left\{\frac{1}{R_{\Omega}}, \frac{1}{K_{\Omega}}\right\} .
$$

While, if $K_{\Omega}=0$ or $K_{\Omega} \neq 0$ with $b \neq 0$ and $R_{J} \leq 1$ then

$$
\Lambda=\max \left\{\frac{1}{R_{\Omega}}, \frac{1}{K_{\Omega}+1}\right\} .
$$

Proof. Let $x_{0} \in \Omega$ such that $R_{\Omega}=\operatorname{dist}\left(x_{0}, \partial \Omega\right)$ and define

$$
v(x)=\frac{\left(R_{\Omega}-\left|x-x_{0}\right|\right)_{+}}{R_{\Omega} .}
$$




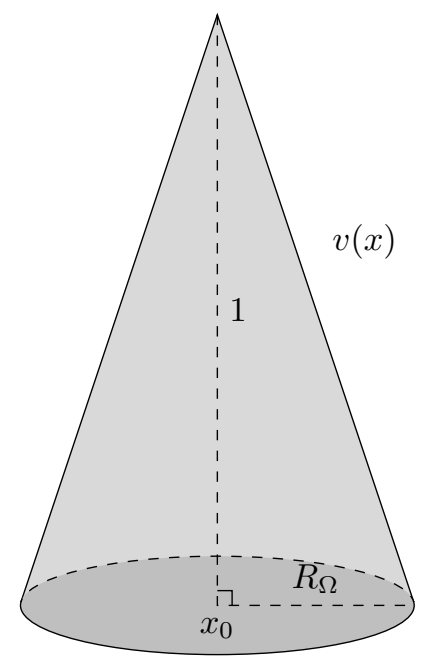

Observe that $v \in W_{0}^{1, \infty}(\Omega)$ and

$$
\begin{gathered}
\|v\|_{\infty}=1, \quad\|\nabla v\|_{\infty}=\frac{1}{R_{\Omega}} \\
{[v]_{J}= \begin{cases}1 & \text { if } R_{J}>R_{\Omega} \\
\frac{R_{J}}{R_{\Omega}} & \text { if } R_{J} \leq R_{\Omega} .\end{cases} }
\end{gathered}
$$

Then

$$
\frac{\max \left\{\|\nabla v\|_{\infty},[v]_{J}\right\}}{\|v\|_{\infty}}= \begin{cases}\max \left\{\frac{1}{R_{\Omega}}, 1\right\} & \text { if } R_{J}>R_{\Omega}, \\ \max \left\{\frac{1}{R_{\Omega}}, \frac{R_{J}}{R_{\Omega}}\right\} & \text { if } R_{J} \leq R_{\Omega} .\end{cases}
$$

Now, we can observe that

- If $K_{\Omega}=0$ then $\frac{1}{K_{\Omega}+1}=1$;

- If $b=0$ then $\frac{1}{K_{\Omega}}=\frac{R_{J}}{R_{\Omega}}$;

- If $K_{\Omega}>0, b>0$ and $R_{J} \leq 1$ then

$$
\frac{1}{K_{\Omega}+1}=\frac{R_{J}}{\left(K_{\Omega}+1\right) R_{J}} \leq \frac{R_{J}}{R_{\Omega}} \leq \frac{1}{R_{\Omega}} .
$$

Then, by Lemma 4.1 and (17), the theorem follows.

To give a complete characterization of $\Lambda$, we have to study the case $K_{\Omega}>0$, $b>0$ and $R_{J}>1$. In this case, we observe the big difference between our nonlocal operator and the $p$-Laplacian and the fractional $p$-Laplacian (see $[18,19,22])$ due to the fact that $\Lambda$ is not achieved by the cone.

Theorem 4.2. Let $K_{\Omega}>0, b>0$ and $R_{J}>1$. If $b \geq 1$ then

$$
\Lambda=\frac{1}{K_{\Omega}+1} \text {. }
$$

Proof. By Lemma 4.1, we have

$$
\Lambda \geq \frac{1}{K_{\Omega}+1}
$$


Therefore, we only need to show the inverse inequality.

Take again $x_{0} \in \Omega$ such that $R_{\Omega}=\operatorname{dist}\left(x_{0}, \partial \Omega\right)$ and we define:

- For $i \in\left\{0, \ldots, K_{\Omega}-1\right\}$

$$
\begin{aligned}
A_{i} & =\left[i R_{J}, i R_{J}+b\right], \quad B_{i}=\left[i R_{J}+b,(i+1) R_{J}\right] \\
h_{i}(t) & =\left(1-\frac{i+\chi_{B_{i}}(t)}{K_{\Omega}+1}\right)-\frac{1}{K_{\Omega}+1} \frac{t-i R_{J}}{b} \chi_{A_{i}}(t) ;
\end{aligned}
$$

- For $i=K_{\Omega}$

$$
I=\left[R_{\Omega}-b, R_{\Omega}\right), \quad h_{K_{\Omega}}(t)=\frac{1}{K_{\Omega}+1}\left(\frac{R_{\Omega}-t}{R-K_{\Omega} R_{J}}\right) \chi_{I}(t) ;
$$

- Finally, we take

$$
h(t)=\sum_{i=0}^{K_{\Omega}} h_{i}(t) \quad \text { and } \quad w(x)=h\left(\left|x-x_{0}\right|\right) .
$$

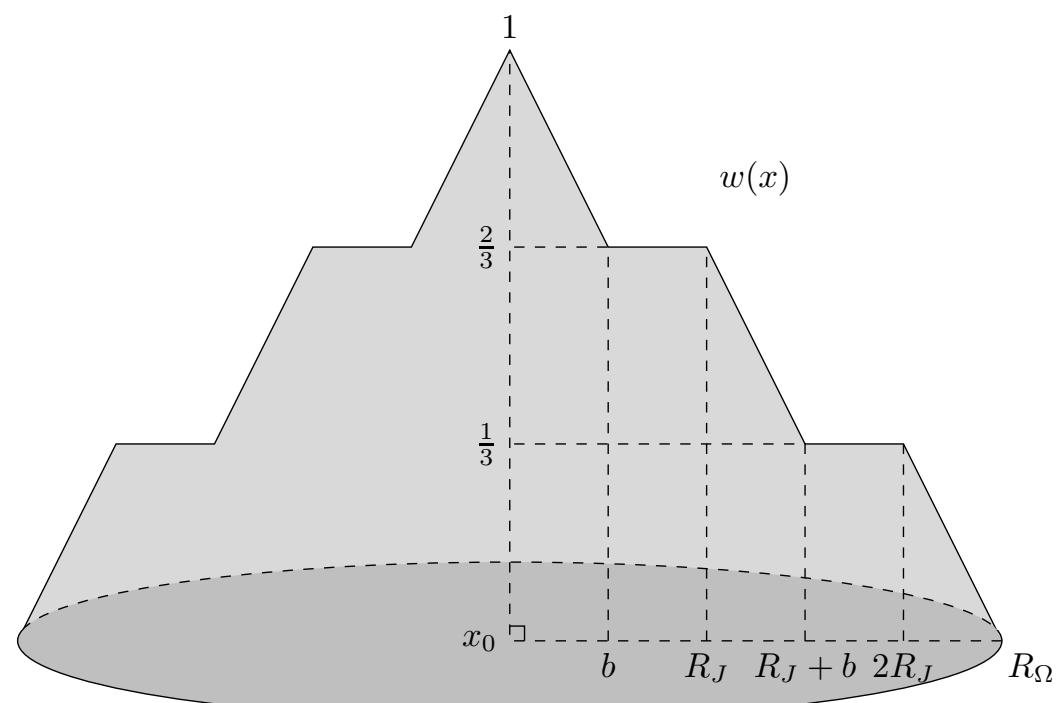

The function $w$ when $K_{\Omega}=2$.

Observe that $w \in W_{0}^{1, \infty}(\Omega),\|w\|_{\infty}=1$,

$$
\|\nabla w\|_{\infty}=\frac{1}{K_{\Omega}+1} \frac{1}{b} \quad \text { and } \quad[w]_{J}=\frac{1}{K_{\Omega}+1} .
$$

Then, if $b \geq 1$, we have

$$
\Lambda \leq \max \left\{\|\nabla w\|_{\infty},[w]_{J}\right\}=\frac{1}{K_{\Omega}+1} .
$$

Theorem 4.3. Let $K_{\Omega}>0, b>0$ and $R_{J}>1$. If $b<1$ then

$$
\Lambda=\frac{1}{K_{\Omega}+b} \text {. }
$$


Proof. Let us observe that as $b<1<R_{J}$ we get

$$
R_{\Omega}+b<R_{\Omega}+1, \quad K_{\Omega}+b<K_{\Omega} R_{J}+b=R_{\Omega}
$$

then by Lemma 4.1

$$
\Lambda \geq \frac{1}{K_{\Omega}+b}
$$

Therefore, we only need to show the inverse inequality.

Take again $x_{0} \in \Omega$ such that $R_{\Omega}=\operatorname{dist}\left(x_{0}, \partial \Omega\right)$ and we define:

- For $i \in\left\{0, \ldots, K_{\Omega}-1\right\}$

$$
\begin{aligned}
& A_{i}=\left[i R_{J}, i R_{J}+b\right], \quad B_{i}=\left[i R_{J}+b,(i+1) R_{J}\right] \quad y_{i}=1-\frac{i}{K_{\Omega}+b} \\
& f(t)= \begin{cases}y_{i}-\frac{1}{K_{\Omega}+b}\left(t-i R_{J}\right) & t \in A_{i}, \\
y_{i}-\frac{b}{K_{\Omega}+b}-\frac{1-b}{\left(K_{\Omega}+b\right)\left(R_{J}-b\right)}\left(t-i R_{J}-b\right) & t \in B_{i} ;\end{cases}
\end{aligned}
$$

- For $i=K_{\Omega}$

$$
f(t)=\frac{b}{K_{\Omega}+b}-\frac{1}{K_{\Omega}+b}\left(t-R_{\Omega}+b\right) \quad t \in\left[R_{\Omega}-b, R_{\Omega}\right) ;
$$

- $z: \mathbb{R}^{N} \rightarrow \mathbb{R}$

$$
z(x)= \begin{cases}f\left(\left|x-x_{0}\right|\right) & \text { if }\left|x-x_{0}\right| \leq R_{\Omega} \\ 0 & \text { if }\left|x-x_{0}\right|>R_{\Omega}\end{cases}
$$

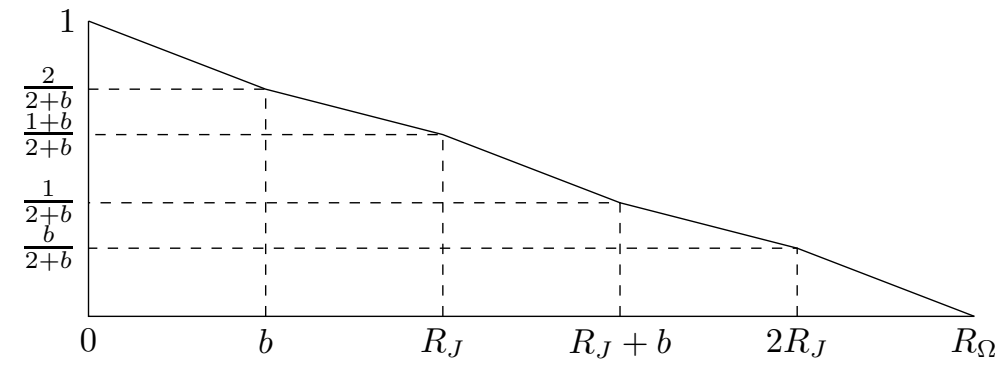

The function $f$ when $K_{\Omega}=2$.

Observe that $z \in W_{0}^{1, \infty}(\Omega),\|z\|_{\infty}=1$,

$$
[z]_{J}=\frac{1}{K_{\Omega}+b}
$$

and

$$
\|\nabla z\|_{\infty}=\max \left\{\frac{1}{K_{\Omega}+b}, \frac{1-b}{\left(K_{\Omega}+b\right)\left(R_{J}-b\right)}\right\}=\frac{1}{K_{\Omega}+b}
$$

because $R_{J}>1$. Thus,

$$
\Lambda \leq \frac{1}{K_{\Omega}+b}
$$

and so finish the proof. 


\section{The LiMIT PROBLEM}

Our last aim is show that $u_{\infty}$ is a viscosity solution of

$$
\begin{cases}\max \left\{M_{1}(u), M_{2}(u)\right\}=0 & \text { in } \Omega \\ u=0 & \text { on } \partial \Omega\end{cases}
$$

where

$$
M_{1}(u(x)):=\min \left\{-\Lambda u(x)-\mathcal{L}_{J, \infty}^{-} u(x),-\mathcal{L}_{J, \infty} u(x),-\mathcal{L}_{\infty, J}^{-} u(x)-|\nabla u(x)|\right\}
$$

and

$$
\begin{gathered}
M_{2}(u(x)):=\min \left\{|\nabla u(x)|-\Lambda u(x),-\Delta_{\infty} u(x),|\nabla u(x)|-\mathcal{L}_{\infty, J}^{+} u(x),\right. \\
\left.|\nabla u(x)|+\mathcal{L}_{\infty, J}^{-} u(x)\right\} .
\end{gathered}
$$

The following result follows from [8, Lemma 6.5]. We include the proof for reader's convenience.

Lemma 5.1. Let $\phi \in C_{0}^{1}(\bar{\Omega})$ extended by zero outside $\Omega$ and $x_{p} \rightarrow x_{0}$ as $p \rightarrow \infty$. Then,

$$
\left(\int_{\mathbb{R}^{N}} J\left(x_{p}-y\right)\left(\phi(y)-\phi\left(x_{p}\right)\right)_{+}^{p-1} d y\right)^{\frac{1}{p-1}} \rightarrow \mathcal{L}_{J, \infty}^{+} \phi\left(x_{0}\right)
$$

and

$$
\left(\int_{\mathbb{R}^{N}} J\left(x_{p}-y\right)\left(\phi(y)-\phi\left(x_{p}\right)\right)_{-}^{p-1} d y\right)^{\frac{1}{p-1}} \rightarrow-\mathcal{L}_{J, \infty}^{-} \phi\left(x_{0}\right) .
$$

Proof. We just examine the first convergence, since the same argument can be used to analyze the second one. Let us define

$$
A_{p}=\int_{\mathbb{R}^{N}} J\left(x_{p}-y\right)\left(\phi(y)-\phi\left(x_{p}\right)\right)_{+}^{p-1} d y \quad \text { and } \quad \mu=\sup _{x_{0}-y \in \operatorname{supp}(J)}\left(\phi(y)-\phi\left(x_{0}\right)\right) \text {. }
$$

The upper bound follows easily

$$
A_{p}^{\frac{1}{p-1}} \leq \sup _{x_{p}-y \in \operatorname{supp}(J)}\left(\phi(y)-\phi\left(x_{p}\right)\right)_{+} \rightarrow \mu \quad \text { as } p \rightarrow \infty
$$

To obtain the lower estimate we assume that $\mu>0$. Observe that as $A_{p} \geq 0$, if $\mu=0$ we are done. Taking $0<t<\mu$ there exists $y_{0} \in B_{R_{J}}\left(x_{0}\right)$ such that $\phi\left(y_{0}\right)-\phi\left(x_{0}\right)>t$. Since $\phi$ is continuous there exist $\delta>0$ such that if $x \in B_{\delta}\left(x_{0}\right)$ and $y \in B_{\delta}\left(y_{0}\right) \subset B_{R_{J}}\left(x_{0}\right)$ then $\phi(y)-\phi(x)>t$. Therefore

$$
A_{p}^{\frac{1}{p-1}} \geq t\left(\int_{B_{\delta}\left(y_{0}\right)} J\left(x_{p}-y\right) d y\right)^{\frac{1}{p-1}} \rightarrow t \quad \text { as } p \rightarrow \infty .
$$

Finally, taking $t \rightarrow \mu$, we obtain the desired result.

Proof of Theorem 1.3. We begin the proof, showing that $u_{\infty}$ is a viscosity supersolution of (18).

Fix a open set $U \subset \Omega$, a point $x_{0} \in U$ and a function $\psi \in C^{2}(U)$ such that $\psi\left(x_{0}\right)=u\left(x_{0}\right), \psi<u$ in $U \backslash\left\{x_{0}\right\}$.

Proceeding as in [19], we can show that there exist $\left\{p_{n}\right\}_{n \in \mathbb{N}}$ and $\left\{x_{n}\right\}_{n \in \mathbb{N}} \subset \Omega$ such that

$$
\begin{gathered}
p_{n} \rightarrow \infty, \quad x_{n} \rightarrow x_{0} \quad \text { as } n \rightarrow \infty, \\
u_{p_{n}} \rightarrow u_{\infty} \quad \text { uniformly in } \bar{\Omega} \quad \text { as } n \rightarrow \infty,
\end{gathered}
$$


and $u_{p_{n}}-\psi$ attains its minimum at point $x_{n}$ for all $n \in \mathbb{N}$.

Since $u_{p_{n}}$ is a viscosity solution of (1) with $\lambda=\lambda_{1}(p)$, we get

$$
\mathcal{L}_{J, p_{n}} w_{n}\left(x_{n}\right) \geq \lambda_{1}\left(p_{n}\right)\left(u_{p_{n}}\left(x_{n}\right)\right)^{p_{n}-1} \quad \forall n \in \mathbb{N}
$$

where

$$
w_{n}(x)= \begin{cases}\psi(x) & \text { if } x \in U, \\ u_{p_{n}}(x) & \text { if } x \in U^{c}\end{cases}
$$

for any $n \in \mathbb{N}$. Thus

$$
\begin{aligned}
& \lambda_{1}\left(p_{n}\right)\left(u_{p_{n}}\right)^{p_{n}-1} \leq \mathcal{L}_{J, p_{n}} w_{n}\left(x_{n}\right) \\
& =-\left[\left|\nabla w_{n}\left(x_{n}\right)\right|^{p_{n}-2} \Delta w_{n}\left(x_{n}\right)+\left(p_{n}-2\right)\left|\nabla w_{n}\left(x_{n}\right)\right|^{p_{n}-4} \Delta_{\infty} w_{n}\left(x_{n}\right)\right] \\
& \quad-2 \int_{\mathbb{R}^{N}} J\left(x_{n}-y\right)\left(w_{n}(y)-w_{n}\left(x_{n}\right)\right)_{+}^{p_{n}-1} d y \\
& \quad+2 \int_{\mathbb{R}^{N}} J\left(x_{n}-y\right)\left(w_{n}(y)-w_{n}\left(x_{n}\right)\right)_{-}^{p_{n}-1} d y
\end{aligned}
$$

Then

$$
\begin{aligned}
& \lambda_{1}\left(p_{n}\right)\left(u_{p_{n}}\left(x_{n}\right)\right)^{p_{n}-1}+2 \int_{\mathbb{R}^{N}} J\left(x_{n}-y\right)\left(w_{n}(y)-w_{n}\left(x_{n}\right)\right)_{+}^{p_{n}-1} d y \\
& \leq- {\left[\left|\nabla w_{n}\left(x_{n}\right)\right|^{p_{n}-2} \Delta w_{n}\left(x_{n}\right)+\left(p_{n}-2\right)\left|\nabla w_{n}\left(x_{n}\right)\right|^{p_{n}-4} \Delta_{\infty} w_{n}\left(x_{n}\right)\right] } \\
&+2 \int_{\mathbb{R}^{N}} J\left(x_{n}-y\right)\left(w_{n}(y)-w_{n}\left(x_{n}\right)\right)_{-}^{p_{n}-1} d y .
\end{aligned}
$$

Suppose that there is a subsequence $\left\{x_{n_{j}}\right\}_{j \in \mathbb{N}}$ such that $x_{j} \rightarrow x_{0}$ as $j \rightarrow \infty$ and

$$
\begin{aligned}
& \int_{\mathbb{R}^{N}} J\left(x_{n_{j}}-y\right)\left(w_{n_{j}}(y)-w_{n_{j}}\left(x_{n_{j}}\right)\right)_{-}^{p_{n_{j}}-1} d y \\
& \geq-\left[\left|\nabla w_{n_{j}}\left(x_{n_{j}}\right)\right|^{p_{n_{j}}-2} \Delta w_{n_{j}}\left(x_{n_{j}}\right)+\left(p_{n_{j}}-2\right)\left|\nabla w_{n_{j}}\left(x_{n_{j}}\right)\right|^{p_{n_{j}}-4} \Delta_{\infty} w_{n_{j}}\left(x_{n_{j}}\right)\right] .
\end{aligned}
$$

Then, using (19), we get

$$
\begin{aligned}
& \left(\int_{\mathbb{R}^{N}} J\left(x_{n_{j}}-y\right)\left(w_{n_{j}}(y)-w_{n_{j}}\left(x_{n_{j}}\right)\right)_{-}^{p_{n_{j}}-1} d y\right)^{\frac{1}{p_{n_{j}}-1}} \\
& \geq\left(\left(p_{n_{j}}-2\right)\left|\nabla w_{n_{j}}\left(x_{n_{j}}\right)\right|^{p_{n_{j}}-4}\right)^{\frac{1}{p_{n_{j}}-1}}\left|\frac{\left|\nabla w_{n_{j}}\left(x_{n_{j}}\right)\right|^{2}}{p_{n_{j}}-2} \Delta w_{n_{j}}\left(x_{n_{j}}\right)+\Delta_{\infty} w_{n_{j}}\left(x_{n_{j}}\right)\right|^{\frac{1}{p_{n_{j}}-1}},
\end{aligned}
$$

and

$$
\begin{aligned}
& \left(\lambda_{1}\left(p_{n_{j}}\right)\left(u_{p_{n_{j}}}\left(x_{n_{j}}\right)\right)^{p_{n_{j}}-1}\right. \\
& \left.\quad+2 \int_{\mathbb{R}^{N}} J\left(x_{n_{j}}-y\right)\left(w_{n_{j}}(y)-w_{n_{j}}\left(x_{n_{j}}\right)\left(x_{n_{j}}\right)\right)_{+}^{p_{n_{j}}-1} d y\right)^{\frac{1}{p_{n_{j}}-1}} \\
& \quad \leq\left(3 \int_{\mathbb{R}^{N}} J\left(x_{n_{j}}-y\right)\left(w_{n_{j}}(y)-w_{n_{j}}\left(x_{n_{j}}\right)\left(x_{n_{j}}\right)\right)_{-}^{p_{n_{j}}-1} d y\right)^{\frac{1}{p_{n_{j}}-1}}
\end{aligned}
$$

for any $j \in \mathbb{N}$. Passing to the limit as $n_{j} \rightarrow \infty$ by Lemma 5.1, we get

$$
\begin{aligned}
& \left|\nabla w\left(x_{0}\right)\right| \leq-\mathcal{L}_{J, \infty}^{-} w\left(x_{0}\right), \\
& \max \left\{\Lambda w\left(x_{0}\right), \mathcal{L}_{J, \infty}^{+} w\left(x_{0}\right)\right\} \leq-\mathcal{L}_{J, \infty}^{-} w\left(x_{0}\right),
\end{aligned}
$$


where

Therefore,

$$
w(x)= \begin{cases}\psi(x) & \text { if } x \in U \\ u_{\infty}(x) & \text { if } x \in U^{c}\end{cases}
$$

$$
\min \left\{-\Lambda w\left(x_{0}\right)-\mathcal{L}_{J, \infty}^{-} w\left(x_{0}\right),-\mathcal{L}_{J, \infty} w\left(x_{0}\right),-\mathcal{L}_{\infty, J}^{-} w\left(x_{0}\right)-\left|\nabla w\left(x_{0}\right)\right|\right\} \geq 0,
$$

that is

$$
M_{1}\left(w\left(x_{0}\right)\right) \geq 0
$$

On the other hand, if the subsequence $\left\{x_{n_{j}}\right\}_{j \in \mathbb{N}}$ does not exist, then there is $n_{0}$ such that

$$
\begin{aligned}
& \int_{\mathbb{R}^{N}} J\left(x_{n}-y\right)\left(w_{n}(y)-w_{n}\left(x_{n}\right)\right)_{-}^{p_{n}-1} d y \\
& \leq-\left[\left|\nabla w_{n}\left(x_{n}\right)\right|^{p_{n}-2} \Delta w_{n_{j}}\left(x_{n}\right)+\left(p_{n}-2\right)\left|\nabla w_{n}\left(x_{n}\right)\right|^{p_{n}-4} \Delta_{\infty} w_{n}\left(x_{n}\right)\right]
\end{aligned}
$$

for all $n \geq n_{0}$. Then, from (19) we get that $\nabla w_{n}\left(x_{n}\right) \neq 0$,

$$
\begin{aligned}
& \frac{\left|\nabla w_{n}\left(x_{n}\right)\right|^{3}}{p_{n}-2}\left(\frac{\left(\int_{\mathbb{R}^{N}} J\left(x_{n}-y\right)\left(w_{n}(y)-w_{n}\left(x_{n}\right)\right)_{-}^{p_{n}-1} d y\right)^{\frac{1}{p_{n-1}}}}{\left|\nabla w_{n}\left(x_{n}\right)\right|}\right)^{p_{n}-1} \\
& \leq-\frac{\left|\nabla w_{n}\left(x_{n}\right)\right|^{2}}{p_{n}-2} \Delta w_{n}\left(x_{n}\right)-\Delta_{\infty} w_{n}\left(x_{n}\right)
\end{aligned}
$$

and

(21)

$$
\begin{aligned}
& \frac{\lambda_{1}\left(p_{n}\right)^{\frac{4}{p_{n}}}\left(u_{p_{n}}\left(x_{n}\right)\right)^{3}}{3\left(p_{n}-2\right)}\left(\frac{\lambda_{1}\left(p_{n}\right)^{\frac{1}{p_{n}}} u_{p_{n}}\left(x_{n}\right)}{\left|\nabla w_{n}\left(x_{n}\right)\right|}\right)^{p_{n}-4} \\
& \quad+\frac{2\left|\nabla w_{n}\left(x_{n}\right)\right|^{3}}{3\left(p_{n}-2\right)}\left(\frac{\left(\int_{\mathbb{R}^{N}} J\left(x_{n}-y\right)\left(w_{n}(y)-w_{n}\left(x_{n}\right)\right)_{+}^{p_{n}-1} d y\right)^{\frac{1}{p_{n}-1}}}{\left|\nabla w_{n}\left(x_{n}\right)\right|}\right)^{p_{n}-1} \\
& \leq-3\left[\frac{\left|\nabla w_{n}\left(x_{n}\right)\right|^{2}}{p_{n}-2} \Delta w_{n}\left(x_{n}\right)+\Delta_{\infty} w_{n}\left(x_{n}\right)\right] .
\end{aligned}
$$

Since in the previous inequalities the right hand side is bounded, we get

$$
\begin{aligned}
\frac{-\mathcal{L}_{\infty, J}^{-} w\left(x_{0}\right)}{\left|\nabla w\left(x_{0}\right)\right|} & \leq 1, \\
\frac{\Lambda w\left(x_{0}\right)}{\left|\nabla w\left(x_{0}\right)\right|} & \leq 1 \\
\frac{-\mathcal{L}_{\infty, J}^{+} w\left(x_{0}\right)}{\left|\nabla w\left(x_{0}\right)\right|} & \leq 1,
\end{aligned}
$$

Therefore, passing to the limit in (21), using again Lemma 5.1 we obtain

$$
\begin{gathered}
\min \left\{\left|\nabla w\left(x_{0}\right)\right|-\Lambda w\left(x_{0}\right),-\Delta_{\infty} w\left(x_{0}\right),\left|\nabla w\left(x_{0}\right)\right|-\mathcal{L}_{\infty, J}^{+} w\left(x_{0}\right),\right. \\
\left.\left|\nabla w\left(x_{0}\right)\right|+\mathcal{L}_{\infty, J}^{-} w\left(x_{0}\right)\right\} \geq 0
\end{gathered}
$$

that is

$$
M_{2}\left(w\left(x_{0}\right)\right) \geq 0
$$


Therefore, by (20) and (22)

$$
\max \left\{M_{1}\left(w\left(x_{0}\right)\right), M_{2}\left(w\left(x_{0}\right)\right)\right\} \geq 0
$$

Then $u_{\infty}$ is a viscosity supersolution of (18).

To finish the proof, we need to show that $u_{\infty}$ is a viscosity subsolution of (18). Fix a open set $U \subset \Omega$, a point $x_{0} \in U$ and a function $\psi \in C^{2}(U)$ such that $\phi\left(x_{0}\right)=u\left(x_{0}\right), \phi>u$ in $U \backslash\left\{x_{0}\right\}$. We want to show that

$$
\max \left\{M_{1}\left(v\left(x_{0}\right)\right), M_{2}\left(v\left(x_{0}\right)\right)\right\} \leq 0
$$

where

$$
v(x)= \begin{cases}\phi(x) & \text { if } x \in U, \\ u_{\infty}(x) & \text { if } x \in U^{c}\end{cases}
$$

Proceeding as in [19], we can show that there exist $\left\{p_{n}\right\}_{n \in \mathbb{N}}$ and $\left\{x_{n}\right\}_{n \in \mathbb{N}} \subset \Omega$ such that

$$
\begin{gathered}
p_{n} \rightarrow \infty, \quad x_{n} \rightarrow x_{0} \quad \text { as } n \rightarrow \infty, \\
u_{p_{n}} \rightarrow u_{\infty} \quad \text { uniformly in } \bar{\Omega} \quad \text { as } n \rightarrow \infty,
\end{gathered}
$$

and $u_{p_{n}}-\phi$ attains its maximum at point $x_{n}$ for all $n \in \mathbb{N}$.

Since $u_{p_{n}}$ is a viscosity solution of (1) with $\lambda=\lambda_{1}(p)$, we get

$$
\mathcal{L}_{J, p_{n}} v_{n}\left(x_{n}\right) \leq \lambda_{1}\left(p_{n}\right)\left(u_{p_{n}}\left(x_{n}\right)\right)^{p_{n}-1} \quad \forall n \in \mathbb{N}
$$

where

for any $n \in \mathbb{N}$. Then

$$
v_{n}(x)= \begin{cases}\phi(x) & \text { if } x \in U \\ u_{p_{n}}(x) & \text { if } x \in U^{c}\end{cases}
$$

$$
\begin{aligned}
& \lambda_{1}\left(p_{n}\right)\left(u_{p_{n}}\left(x_{n}\right)\right)^{p_{n}-1}+2 \int_{\mathbb{R}^{N}} J\left(x_{n}-y\right)\left(v_{n}(y)-v_{n}\left(x_{n}\right)\right)_{+}^{p_{n}-1} d y \\
& \geq- {\left[\left|\nabla v_{n}\left(x_{n}\right)\right|^{p_{n}-2} \Delta v_{n}\left(x_{n}\right)+\left(p_{n}-2\right)\left|\nabla v_{n}\left(x_{n}\right)\right|^{p_{n}-4} \Delta_{\infty} v_{n}\left(x_{n}\right)\right] } \\
& \quad+2 \int_{\mathbb{R}^{N}} J\left(x_{n}-y\right)\left(v_{n}(y)-v_{n}\left(x_{n}\right)\right)_{-}^{p_{n}-1} d y,
\end{aligned}
$$

and therefore

$$
\begin{aligned}
& \left(\lambda_{1}\left(p_{n}\right)\left(v_{p_{n}}\left(x_{n}\right)\right)^{p_{n}-1}+2 \int_{\mathbb{R}^{N}} J\left(x_{n}-y\right)\left(v_{n}(y)-v_{n}\left(x_{n}\right)\right)_{+}^{p_{n}-1} d y\right. \\
& \left.\quad+\left(p_{n}-2\right)\left|\nabla v_{n}\left(x_{n}\right)\right|^{p_{n}-4}\left|\frac{\left|\nabla v_{n}\left(x_{n}\right)\right|^{2}}{p_{n}-2} \Delta v_{n}\left(x_{n}\right)+\Delta_{\infty} v_{n}\left(x_{n}\right)\right|\right)^{\frac{1}{p_{n}-1}} \\
& \geq\left(2 \int_{\mathbb{R}^{N}} J\left(x_{n}-y\right)\left(v_{n}(y)-v_{n}\left(x_{n}\right)\right)_{-}^{p_{n}-1} d y\right)^{\frac{1}{p_{n}-1}} .
\end{aligned}
$$

Thus, passing to the limit using again Lemma 5.1 we obtain

$$
\max \left\{\Lambda v\left(x_{0}\right), \mathcal{L}_{\infty, J}^{+} v\left(x_{0}\right),\left|\nabla v\left(x_{0}\right)\right|\right\} \geq-\mathcal{L}_{\infty, J}^{-} v\left(x_{0}\right) .
$$

Hence

$$
0 \geq \min \left\{-\Lambda v\left(x_{0}\right)-\mathcal{L}_{\infty, J}^{-} v\left(x_{0}\right),-\mathcal{L}_{\infty, J} v\left(x_{0}\right),-\left|\nabla v\left(x_{0}\right)\right|-\mathcal{L}_{\infty, J}^{-} v\left(x_{0}\right)\right\},
$$

that is

$$
M_{1}\left(v\left(x_{0}\right)\right) \leq 0
$$


On the other hand, if $\left|\nabla v\left(x_{0}\right)\right|=0$ then

$$
M_{2}\left(v\left(x_{0}\right)\right) \leq 0 .
$$

We now assume that $\left|\nabla v\left(x_{0}\right)\right|>0$ and

(26) $\min \left\{\left|\nabla v\left(x_{0}\right)\right|-\Lambda v\left(x_{0}\right),\left|\nabla v\left(x_{0}\right)\right|-\mathcal{L}_{\infty, J}^{+} v\left(x_{0}\right),\left|\nabla v\left(x_{0}\right)\right|+\mathcal{L}_{\infty, J}^{-} v\left(x_{0}\right)\right\}>0$.

By (23), we get

$$
\begin{aligned}
& \frac{\lambda_{1}\left(p_{n}\right)^{\frac{4}{p_{n}}}\left(u_{p_{n}}\left(x_{n}\right)\right)^{3}}{3\left(p_{n}-2\right)}\left(\frac{\lambda_{1}\left(p_{n}\right)^{\frac{1}{p_{n}}} u_{p_{n}}\left(x_{n}\right)}{\left|\nabla v_{n}\left(x_{n}\right)\right|}\right)^{p_{n}-4} \\
& \quad+\frac{2\left|\nabla v_{n}\left(x_{n}\right)\right|^{3}}{3\left(p_{n}-2\right)}\left(\frac{\left(\int_{\mathbb{R}^{N}} J\left(x_{n}-y\right)\left(v_{n}(y)-v_{n}\left(x_{n}\right)\right)_{+}^{p_{n}-1} d y\right)^{\frac{1}{p_{n}-1}}}{\left|\nabla v_{n}\left(x_{n}\right)\right|}\right)^{p_{n}-1} \\
& \geq-\left[\frac{\left|\nabla v_{n}\left(x_{n}\right)\right|^{2}}{p_{n}-2} \Delta v_{n}\left(x_{n}\right)+\Delta_{\infty} v_{n}\left(x_{n}\right)\right] .
\end{aligned}
$$

Thus, passing to the limit and using (26) and Lemma 5.1 we get

$$
0 \geq-\Delta_{\infty} v\left(x_{0}\right)
$$

Therefore

$$
M_{2}\left(v\left(x_{0}\right)\right) \leq 0 .
$$

Finally by (24),(25), and (27), we have that

$$
\max \left\{M_{1}\left(v\left(x_{0}\right)\right), M_{2}\left(v\left(x_{0}\right)\right)\right\} \leq 0
$$

and therefore $u_{\infty}$ is a viscosity subsolution of (18).

\section{ACKNOWLEDGEMENTS}

LDP and JDR are partially supported by PIP GI No 11220150100036CO. CONICET (Argentina). JDR is also supported by MINECO MTM2015-70227-P (Spain) and Subsidio 20020160100155BA. UBACyT (Argentina). RF is supported by the Spanish project MTM2017-87596-P.

\section{REFERENCES}

[1] W. Allegretto and Y. X. Huang, A Picones identity for the p-lapacian and applications. Non-linear Analysis, Theory, Methods \& Applications, 32 (1989), no. 7, 819-830.

[2] S. Amghibech, On the discrete version of Picone's identity. Discrete Appl. Math. 156 (2008), no. $1,1-10$

[3] F. Andreu-Vaillo, J. M. Mazon, J. D. Rossi and J. J. Toledo-Melero. Nonlocal Diffusion Problems. American Mathematical Society. Mathematical Surveys and Monographs 2010. Vol. 165.

[4] F. Andreu, J. M. Mazon, J. D. Rossi And J. Toledo. The limit as $p \rightarrow \infty$ in a nonlocal $p$-Laplacian evolution equation. A nonlocal approximation of a model for sandpiles. Calc. Var. Partial Diff. Eq. 35(3), (2009), 279-316.

[5] F. Andreu, J. M. Mazon, J. D. Rossi and J. Toledo. A nonlocal p-Laplacian evolution equation with non homogeneous Dirichlet boundary conditions. SIAM J. Math. Anal. 40(5), (2009), 1815-1851.

[6] L. Brasco, E. Parini and M. Squassina, Stability of variational eigenvalues for the fractional p-Laplacian, Discr. Cont. Dyn. Sys., 36, (2016), 1813-1845.

[7] L. Caffarelli, Nonlocal equations, drifts and games. In: Nonlinear Partial Differential Equations, Springer, Berlin (2012), 37-52.

[8] A. Chambolle, E. Lindgren and R. Monneau. A Hölder Infinity Laplacian. ESAiM Control Optim. Calc. Var. DOI: 10.1051/cocv/2011182 
[9] L. Del Pezzo And A. QuaAs, Global bifurcation for fractional p-Laplacian and an application. Z. Anal. Anwend. 35 (2016), no. 4, 411-447.

[10] L. Del Pezzo, J. Fernandez Bonder And L. Lopez Rios, An optimization problem for the first eigenvalue of the p-fractional Laplacian. To appear in Math. Nachr.

[11] L. Del Pezzo And J.D. Rossi, Eigenvalues for a nonlocal pseudo p-Laplacian. Discrete and Contin. Dynam. Systems 36, No 12 (2016), 6737-6765.

[12] E. Di Nezza, G. Palatucci and E. Valdinoci, Hitchhiker's guide to the fractional Sobolev spaces, Bull. Sci. math., 136, (2012), 521-573.

[13] M. Farcaseanu, M. Mihailescu, D. Stancu-Dumitru, Perturbed fractional eigenvalue problems. Discrete and Contin. Dynam. Systems - A 37, No 12 (2017), 6243-6255.

[14] G. Franzina and G. Palatucci, Fractional p-eigenvalues. Riv. Math. Univ. Parma (N.S.) 5, (2014), no. 2, 373-386.

[15] J. Garcia-Azorero And I. Peral. Existence and nonuniqueness for the p-Laplacian: Nonlinear eigenvalues, Comm. Partial Differential Equations, 12, (1987), 1389-1430.

[16] J. Garcia-Azorero AND I. Peral, Multiplicity of solutions for elliptic problems with critical exponent or with a nonsymmetric term, Trans. Amer. Math. Soc., 323, (1991), 877-895.

[17] V. Julin And P. Juutinen, A new proof for the equivalence of weak and viscosity solutions for the p-Laplace equation. Comm. Partial Differential Equations 37 (2012), no. 5, 934-946.

[18] P. JuUtinen AND P. LindQvist, On the higher eigenvalues for the $\infty$-eigenvalue problem. Calc. Var. Partial Differential Equations 23 (2005), no. 2, 169-192.

[19] P. Juutinen, P. Lindqvist and J. J. Manfredi, The $\infty$-eigenvalue problem. Arch. Ration. Mech. Anal. 148 (1999), no. 2, 89-105.

[20] H. JyLhA, An optimal transportation problem related to the limits of solutions of local and nonlocal p-Laplace-type problems, Rev. Mat. Complutense, 28, (2015), 85-121.

[21] G. M. Lieberman, Boundary regularity for solutions of degenerate elliptic equations. Nonlinear Anal. 12 (1988), no. 11, 1203-1219.

[22] E. Lindgren and P. Lindqvist, Fractional eigenvalues. Calc. Var. Partial Differential Equations 49 (2014), no. 1-2, 795-826.

[23] P. Lindqvist. Lectures on the p-harmonic equation. Summerschool; 2016-07-18 - 2016-07-20 NTNU.

[24] E. PARINI, An introduction to the Cheeger problem, Surv. Math. Appl. 6 (2011), 9-21.

[25] J. Simon, Régularité de la solution d'une équation non linéaire dans $\mathbb{R}^{n}$. Journées d'Analyse Non Linéaire (Proc. Conf., Besançon, 1977), pp. 205-227, Lecture Notes in Math., 665, Springer, Berlin, 1978.

Leandro M. Del Pezzo

CONICET AND UTDT

Departamento de Matemáticas y Estadística

Universidad Torcuato Di Tella

Av. Figueroa Alcorta 7350 (C1428BCW)

Buenos Aires, ARGEntina.

E-mail address: ldelpezzo@utdt.edu

$U R L:$ http://cms.dm.uba.ar/Members/ldpezzo/

RAÚl FERREIRA

Departamento de Matemática Aplicada,

Fac. De C.C. Químicas, U. Complutense de Madrid,

28040, MAdrid, SPAIN.

E-mail address: raul_ferreira@mat.ucm.es

Julio D. Rossi

COniCET and Departamento de Matemática, FCEyn,

Universidad de Buenos Aires,

Pabellon I, Ciudad Universitaria (C1428BCW),

Buenos Aires, Argentina.

E-mail address: jrossi@dm.uba.ar

$U R L:$ http://mate.dm.uba.ar/ jrossi/ 